\title{
Identification and Functional Annotation of Genome-Wide ER-Regulated Genes in Breast Cancer Based on ChIP-Seq Data
}

\author{
Min Ding, ${ }^{1,2}$ Haiyun Wang, ${ }^{2}$ Jiajia Chen, ${ }^{3}$ Bairong Shen, ${ }^{3}$ and Zhonghua $\mathrm{Xu}^{4}$ \\ ${ }^{1}$ Department of Viral and Gene Therapy, Eastern Hepatobiliary Surgery Hospital, Second Military Medical University, \\ Shanghai 200438, China \\ ${ }^{2}$ School of Life science and Technology, Tongji University, Shanghai 200092, China \\ ${ }^{3}$ Center for Systems Biology, Soochow University, Suzhou Jiangsu 215006, China \\ ${ }^{4}$ Department of Cardiothoracic Surgery, Second Affiliated Hospital of Soochow University, Suzhou Jiangsu 215004, China
}

Correspondence should be addressed to Zhonghua Xu, drxuzh@sohu.com

Received 1 November 2012; Accepted 18 December 2012

Academic Editor: Hong-Bin Shen

Copyright ( $) 2012$ Min Ding et al. This is an open access article distributed under the Creative Commons Attribution License, which permits unrestricted use, distribution, and reproduction in any medium, provided the original work is properly cited.

\begin{abstract}
Estrogen receptor (ER) is a crucial molecule symbol of breast cancer. Molecular interactions between ER complexes and DNA regulate the expression of genes responsible for cancer cell phenotypes. However, the positions and mechanisms of the ER binding with downstream gene targets are far from being fully understood. ChIP-Seq is an important assay for the genome-wide study of protein-DNA interactions. In this paper, we explored the genome-wide chromatin localization of ER-DNA binding regions by analyzing ChIP-Seq data from MCF-7 breast cancer cell line. By integrating three peak detection algorithms and two datasets, we localized 933 ER binding sites, 92\% among which were located far away from promoters, suggesting long-range control by ER. Moreover, 489 genes in the vicinity of ER binding sites were identified as estrogen response elements by comparison with expression data. In addition, 836 single nucleotide polymorphisms (SNPs) in or near 157 ER-regulated genes were found in the vicinity of ER binding sites. Furthermore, we annotated the function of the nearest-neighbor genes of these binding sites using Gene Ontology (GO), KEGG, and GeneGo pathway databases. The results revealed novel ER-regulated genes pathways for further experimental validation. ER was found to affect every developed stage of breast cancer by regulating genes related to the development, progression, and metastasis. This study provides a deeper understanding of the regulatory mechanisms of ER and its associated genes.
\end{abstract}

\section{Introduction}

Breast cancer is a complex disease with high occurrence. It involves a wide range of pathological entities with diverse clinical courses. Gene and protein expression have been extensively profiled in different subtypes of breast cancer [1]. Growth of human breast cells is closely regulated by hormone receptors. Estrogen receptor (ER), a hormonal transcription factor, plays a critical role in the development of breast cancer. Combined with estrogen, it regulates the expression of multiple genes. Studies have found that ERpositive and ER-negative breast cancers are fundamentally different [2]. The outcome of hormone receptor positive tumors is better than hormone receptor negative tumors [3]. Thus, the identification of ER target genes may reveal critical biomarkers for cancer aggressiveness and is therefore crucial to understanding the global molecular mechanisms of ER in breast cancer. To identify direct target genes of $\mathrm{ER}$, it is necessary to map the ER binding sites across the genome. ChIP-Seq is an effective technology for the genomewide localization of histone modification and transcription factor binding sites. It enables researchers to fully understand many biological processes and disease states, including transcriptional regulation of ES cells, tissue samples, and cancer cells.

Several previous studies have been dedicated to ERregulated genes and their function in breast cancer cell line $[4,5]$. However, most studies lacked the comprehensive and genome-wide view and failed to perform an integrated analysis. In this study, we combined ChIP-Seq and microarray 
TABle 1: The CHIP-Seq datasets.

\begin{tabular}{llll}
\hline Dataset & Platform & Cell line & Sample information \\
\hline \multirow{2}{*}{ GSE19013 } & \multirow{2}{*}{ Illumina } & \multirow{2}{*}{ MCF-7 } & Ethanol treated \\
& & & E2-treated \\
GSE14664 & Illumina & \multirow{2}{*}{ MCF-7 } & ER_minus_ligand \\
& & & ER_E2 \\
\hline
\end{tabular}

datasets to analyze the ER-regulated genes in the MCF-7 breast cancer cell line. The molecular mechanisms of ER were fully studied, including binding sites, motif, regulated genes, related single nucleotide polymorphisms (SNPs) and functional annotation. The process of this analysis was illustrated in Figure 1.

\section{Materials and Methods}

2.1. Datasets. The breast cancer associated ChIP-Seq datasets were extracted from Gene Expression Omnibus (GEO): GSE19013 [6] and GSE14664 [7]. Both datasets can be used to survey genome-wide binding of estrogen receptor (ER) in the MCF-7 breast cancer cell line. Control sample was incorporated for the genomic peak finding of ER. (See Table 1 for details.)

2.2. Chip-Seq Analysis. Bowtie [8] was selected to align sequence tags to human genome. Bowtie is an ultrafast and best short-read aligner. It is suitable for sets of short reads where many reads have at least one good and valid alignment, many reads with relatively high quality, and the number of alignment reported per read is small (closed to 1). ChIP-seq datasets we used were satisfied these criteria. In the analysis, tags were selected using the criterion that alignments had no more than 2 mismatches in the first 35 bases on the high quality end of the read, and the sum of the quality values at all mismatched positions could not exceed 70 .

Peak detection algorithm is crucial to the analysis of ChIP-Seq dataset. Currently, several tools are available to identify genome-wide binding sites of transcription factors, such as FindPeaks [9], F-Seq [10], CisGenome [11], MACS [12], SISSRs [13], and QuEST [14]. These different methods have their own advantages and disadvantages, although they act in a similar manner. Table 2 showed an overview of the characteristics of these algorithms. ChIP-Seq data has regional biases because of sequencing and mapping biases, chromatin structure, and genome copy number variations [15]. It is believed that more robust ChIP-Seq peak predictions can be obtained by matching control samples [12]. In order to get more stable result, three tools, CisGenome, MACS, and QuEST, were used to identify the binding sites of ER in this study. All the three tools systematically used control samples to guide peak finding and calculate the FDR (False Discovery Rate) value of peaks.

Additionally, MEME program [16] was employed for de novo motif search, keeping default options (minimum width: 6, maximum width: 50 , motifs to find: 3 , and minimum sites: $\geq 2)$. For each site, statistical significance $(P$ value) gives the probability of a random string having the same match score or higher. And a criterion of $P$-value $<0.01$ was used here.

2.3. Expression and SNP Analysis. Expression analysis was performed using the same package [17, 18]. Differentially expressed genes were selected based on the q-value less than $1 \%$.

Using the table SNP (131) (dbSNP build 131) [19] in UCSC (http://genome.ucsc.edu/), we identified SNPs near the ER binding sites. The SNPs with at least one mapping in the regions were selected.

2.4. Functional Annotation. Three functional annotation systems, the Gene Ontology (GO) categories [20], canonical KEGG Pathway Maps [21], and commercial software MetaCore-GeneGo Pathway Maps, were used to perform the enrichment analysis for gene function.

Enrichment of GO categories was determined with the Gene Ontology Tree Machine (GOTM) [22], using Hypergeometric test, Multiple test adjustment $(\mathrm{BH})$, and a $P$-value cut-off of 0.01 . WebGestalt (WEB-based GEne SeT AnaLysis Toolkit) [23] (http://bioinfo .vanderbilt.edu/webgestalt/option.php) was used for enrichment of KEGG Pathway. Hypergeometric test, Multiple test adjustment $(\mathrm{BH})$, and a $P$-value cut-off of 0.01 were also used as criterion. MetaCore-GeneGo is a commercial software which offers gene expression pathway analysis and bioinformatics solutions for systems biology research and development. Hypergeometric intersection was used to estimate $P$-value, the lower $P$-value means higher relevance. $P$-value $<0.01$ and FDR $<0.05$ were used as criterion.

\section{Results and Discussion}

3.1. ChIP-Seq Analysis Mapped ER Binding Sites across the Human Genome. Using ChIP-Seq datasets, we identified the global ER binding sites. Sequence tags were firstly aligned to human genome assembly (UCSC, hg19) using Bowtie. Three ChIP-Seq peak calling programs, CisGenome, MACS, and QuEST, were selected to identify the enriched binding peaks. Using a false discovery rate of $0.01,933 \mathrm{ER}$ binding peaks were revealed by all the three tools in both datasets (Table 3 ). There were differences among the predicted results using different methods in both two datasets (Figure 2). The calculated FDR value was not only related to different methods, but also influenced by datasets. The overlapped binding sites seemed to be more robust, with $84.9 \%$ having FDR value less than 0.005 in all methods and datasets. These binding sites were used for the following analysis. Firstly, we compared these binding sites with two published studies by Welboren et al. [7] and $\mathrm{Hu}$ et al. [6]. Our results showed a substantial overlap with the two studies (77.8 and $78.5 \%$, resp.). Also, 719 binding sites, which were shared by all three studies, were likely to be more reliable. The presence of consensus sequence motifs in the ER binding sites was also examined. De novo motif search using the MEME program 
TABle 2: An overview of the characteristics of different Chip-Seq peak detection algorithm.

\begin{tabular}{|c|c|c|c|c|}
\hline Algorithm & Profile & Background model & Control sample & Use control to compute FDR \\
\hline F-Seq & Kernel density estimation (KDE) & & $\sqrt{ }$ & \\
\hline FindPeaks & Aggregation of overlapped tags & Monte Carlo & & \\
\hline SISSRs & Window scan & Poisson & $\sqrt{ }$ & \\
\hline QuEST & Kernel density estimation (KDE) & & $\sqrt{ }$ & $\sqrt{ }$ \\
\hline MACS & Tags shifted then window scan & dynamic Poisson & $\sqrt{ }$ & $\sqrt{ }$ \\
\hline CisGenome & Strand-specific window scan & Negative binomial & $\sqrt{ }$ & $\sqrt{ }$ \\
\hline
\end{tabular}

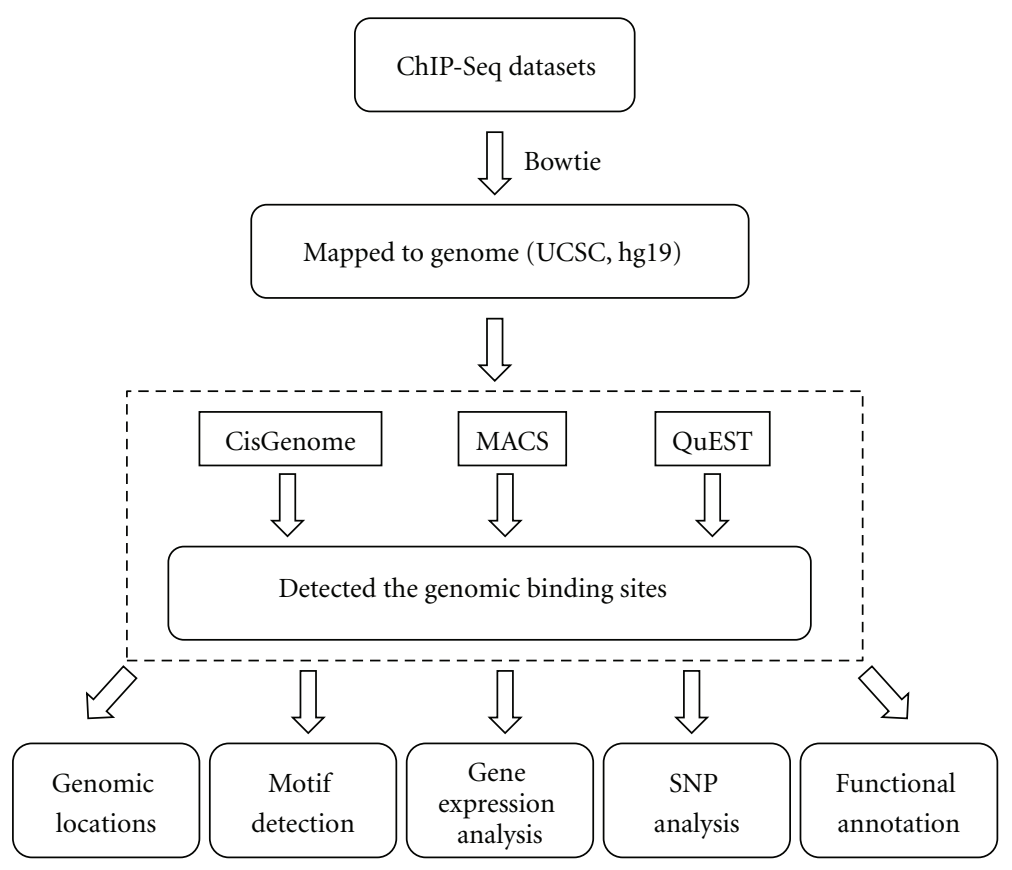

Figure 1: The ChIP-Seq data analyzing pipeline.

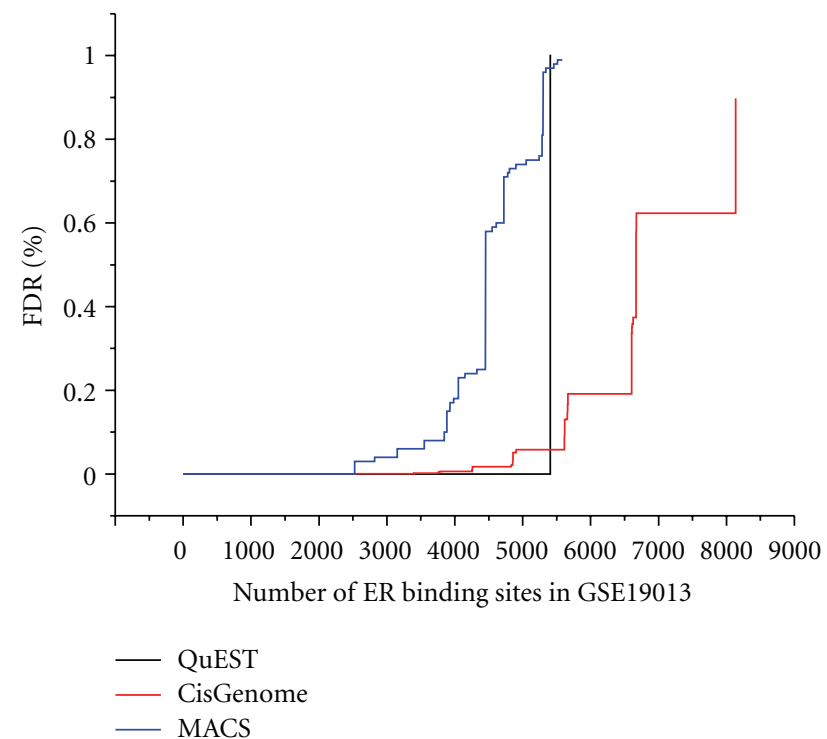

(a)

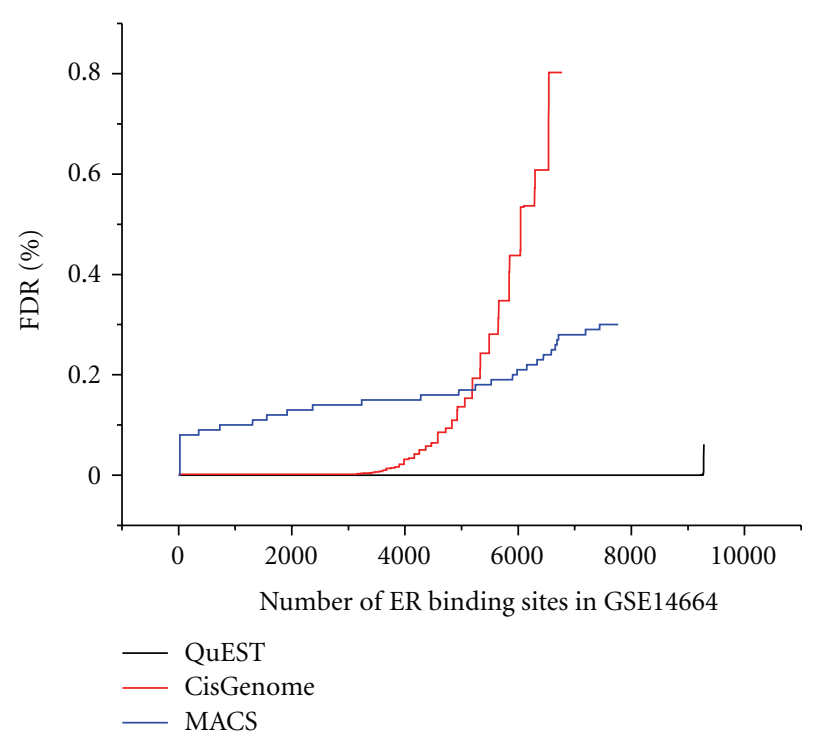

(b)

FIgUre 2: Comparison of QuEST, CisGenome, and MACS predicted result. (a) The FDR value in the dataset of GSE19013. (b) The FDR value in the dataset of GSE14664. 


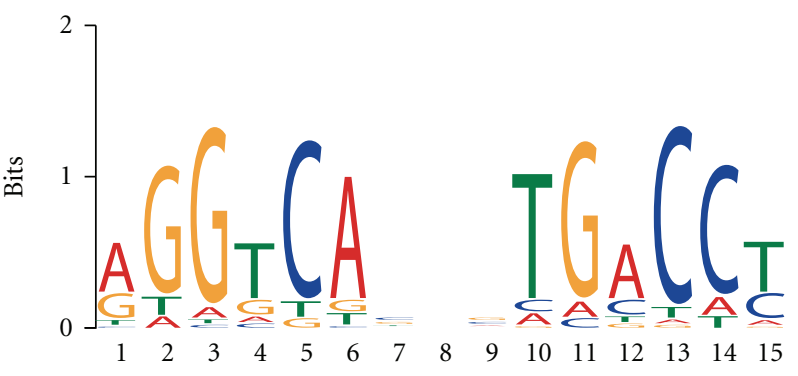

(a)

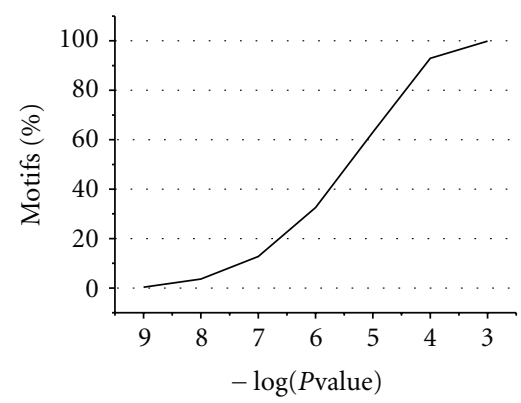

(b)

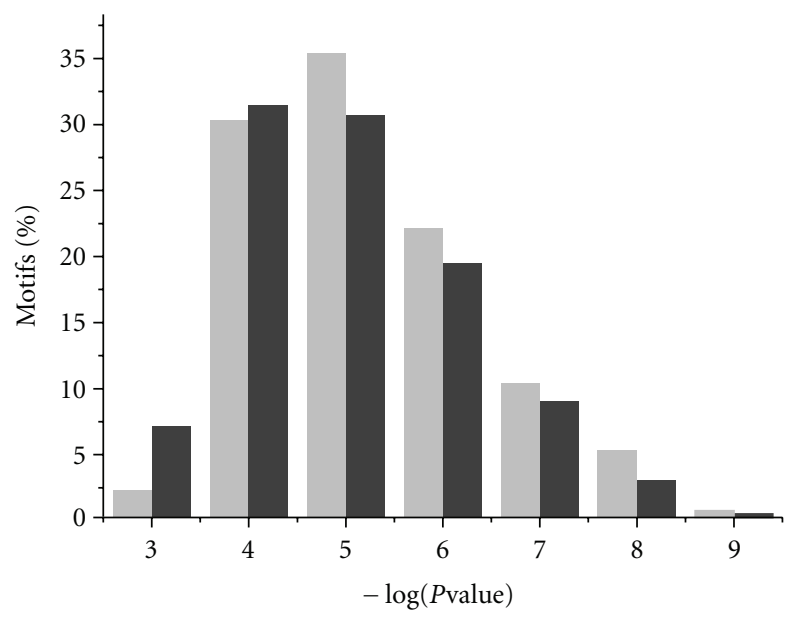

Published

New identified

(c)

FIGURE 3: The genomic binding sites of ER. (a) The consensus motif identified in the ERE binding sites. De novo motif search was performed using the MEME program. (b) The percentage of occurrences of ERE motifs in ER binding sites. (c) Comparison of the occurrences of ERE motifs between published and newly identified binding sites.

TABLE 3: Number of ER binding sites identified by three ChIP-Seq peak calling programs $(F D R<0.01)$.

\begin{tabular}{|c|c|c|c|c|c|}
\hline \multirow[t]{2}{*}{ Dataset } & \multicolumn{3}{|c|}{ Number of ER binding sites } & \multicolumn{2}{|c|}{ Number of overlaped sites } \\
\hline & CisGenome & MACS & QuEST & & \\
\hline GSE19013 & 8137 & 5583 & 5418 & 2019 & 933 \\
\hline GSE14664 & 6773 & 7765 & 9280 & 5061 & נסני \\
\hline
\end{tabular}

[16] identified a refined ERE motif that was markedly similar to the canonical ERE (Figure 3(a)). Almost all of the ER binding sites contained one or more ERE motif $(P$-value $<0.01$ ) (Figure 3(b)). Both published and newly identified binding sites contained at least one ERE motif (Figure 3(c)).

Furthermore, we examined the location of ER enrichment sites relativer to the nearest-neighbor genes. The result was shown in Figure 4(a). Only 8\% (72) of the peaks occured within gene promoters (defined here as within $5 \mathrm{~kb}$ upstream of $5^{\prime}$ to TSS). Also, $34 \%$ (317) of the peaks resided in intragenic sites, including $1 \%(10)$ in the $3^{\prime} \mathrm{UTR}, 9 \%(81)$ in the $5^{\prime} \mathrm{UTR}, 2 \%$ (20) in the exon, and $22 \%$ (206) in the intron. The occupancy of enhancer ( $>5 \mathrm{~kb}$ away $5^{\prime}$ to TSS) was $35 \%$ (332). According to Figure 4(b), the peaks occurred most frequently between $-10 \mathrm{~kb}$ to $-100 \mathrm{~kb},+10 \mathrm{~kb}$ to $+100 \mathrm{~kb}$, with $+10 \mathrm{~kb}$ to $+100 \mathrm{~kb}$ being the highest. A further insight into the peaks within $+10 \mathrm{~kb}$ to $+100 \mathrm{~kb}$ showed that peaks were preferably located within the regions spanning from $+10 \mathrm{~kb}$ to $+40 \mathrm{~kb}$ (Figure $4(\mathrm{c})$ ).

3.2. Using Gene Expression Data to Confirm the ER Binding Sites. In order to determine the specific gene responses corresponding to ER in MCF-7 cells, we compared the nearest-neighbor genes of ER binding sites to the published studies examining differentially expressed genes between ER+ and ER - breast tumors. We used the 3 studies in Table 4 for the gene expression analysis. Differentially expressed genes were selected based on a $q$-value cut-off of less 
TABLE 4: Breast cancer gene expression dataset and differently expressed genes number $(q$-value $<1 \%)$.

\begin{tabular}{|c|c|c|c|c|c|c|}
\hline \multirow{2}{*}{ Author } & \multirow{2}{*}{ Journal } & \multirow{2}{*}{ Array type } & \multirow{2}{*}{$\begin{array}{l}\text { Sample } N \\
\text { ER+ }\end{array}$} & \multirow{2}{*}{$\begin{array}{l}\text { Sample } N \\
\text { ER- }\end{array}$} & \multicolumn{2}{|c|}{ Differently expressed genes } \\
\hline & & & & & Upregulated & Downregulated \\
\hline Graham et al. [24] & Clin Cancer Res & Affy & 15 & 15 & 709 & 333 \\
\hline Wang et al. [25] & Lancet & Affy & 209 & 77 & 2081 & 2537 \\
\hline Lu et al. [26] & $\begin{array}{c}\text { Breast Cancer Res } \\
\text { Treat }\end{array}$ & Affy & 76 & 53 & 5136 & 5445 \\
\hline & & All & & & 5692 & 6101 \\
\hline
\end{tabular}

than $1 \%$ using a stringent statistical analysis method. We identified 5692 and 6101 up- and downregulated genes. When combined with the nearest-neighbor genes of ER binding sites, 289 up-regulated genes and 198 downregulated genes were associated with the ER binding sites (see additional file 1, Supplementary Material available online at doi:10.1155/2012/568950). Among these genes, 33 upregulated genes and 11 downregulated genes were also identified by published ChIP-PET analysis [27].

Our analysis found that more binding sites were associated with ER up-regulated genes $(60 \%)$ compared to down-regulated genes (40\%), indicating that ER was more frequently involved in the direct regulation of up-regulated genes. We also examined the location of ER binding sites in up-regulated and down-regulated genes. As shown in Figure 5, both the up- and down-regulated genes occurred most frequently between $-10 \mathrm{~kb}$ to $-100 \mathrm{~kb},+10 \mathrm{~kb}$ to +100 $\mathrm{kb}$, which verified the long-range control mode of ER factor.

\subsection{SNPs Occurred near the ER Binding Sites. Current studies} have shown that the breast cancer risks are associated with commonly occurring single nucleotide polymorphisms (SNPs) [28-32]. The table SNP (131) (dbSNP build 131) in UCSC (http://genome.ucsc.edu/) was used to identify SNPs near the ER binding sites. A total of 2694 SNP loci were found and subsequently annotated using dbSNP in NCBI.

Compared with the differently expressed gene set in the vicinity of ER binding sites, 836 SNPs in or near 157 ERregulated genes were identified (see additional file 2). Most of the SNPs (94.5\%) were located in intron and untranslated regions. Only 5.5\% were located in the regions of near-gene, coding-synon, missense, and frameshift. These SNPs might have close relationship with breast cancer.

3.4. Functional Annotation of ER Binding Sites. To identify the biological processes and pathways altered by ER, we employed three functional annotation systems, the Gene Ontology (GO) categories [20], canonical KEGG Pathway Maps [21], and commercial software MetaCore-GeneGo Pathway Maps, to perform the enrichment analysis for gene function.

To gain an overview of the biological processes in which the nearest-neighbor genes of ER binding sites reside, we firstly performed gene set enrichment analysis using Gene Ontology database. Statistically significant (Hypergeometric test, $P$-value $<0.01)$ enriched GO terms were identified using the web tool GOTM (Gene Ontology Tree Machine)
[22]. The Gene Ontology Directed Acyclic Graph for the nearest-neighbor genes generated by GOTM was presented in Figure 6. The terms with red color were significantly enriched. In terms of biological process, negative regulation of biological process and cellular process, cellular component movement, and regulation of localization and locomotion, structure and system development were significantly enriched. Furthermore, whether differently expressed or not, genes were mostly associated with biological regulation and metabolic process in biological process terms, protein binding in molecular function terms, and membrane in cellular component terms (each term included more than 100 genes). Gene functions for all the nearest-neighbor genes were summarized in Table 5.

The KEGG Pathway database (posted on May 23, 2011) was used to identify functional modules regulated by ER. Seventeen significantly enriched pathways $(P$-value $<0.01)$ were revealed (Table 6 ). In these pathways, most genes were also differentially expressed between ER+ and ER- tumors. Pathways in cancer, focal adhesion, axon guidance, regulation of actin cytoskeleton, and MAPK signaling pathway ranked among the most enriched pathways. The top enriched maps, such as focal adhesion pathway and MAPK signaling pathway, were reported to be related with ER in breast cancer. High expression of focal adhesion kinase had been reported to be related to cancer progression of breast. And tumors with high expression of focal adhesion kinase lack ER and PR [33]. It was also reported that hyperactivation of MAPK could repress the ER expression in breast tumors [34]. Pathways in cancer were the top enriched KEGG pathway. The abnormal expression of some genes occurred in several types of cancer [35-37]. Axon guidance pathway played important roles in cancers. Axon guidance molecules might control the development, migration, and invasion of cancer cells [38]. Regulation of actin cytoskeleton was related to cancer cell migration and invasion [39]. This indicated the crucial role of ER in the development, migration, and invasion of breast cancer.

GeneGo was also used to perform the pathway analysis. Ten pathways were found to be significantly enriched with $P$-value $<0.01$ and FDR $<0.05$ (Table 7). The result showed that ER binding sites were enriched in breast cancer related pathways. Among the top five maps, development_prolactin receptor signaling and development_glucocorticoid receptor signaling had been reported to associate with ER [40, 41]. development_ligand-independent activation of ESR1 and ESR2 was another enriched map which might have close 
TABLE 5: The comparison of top enriched GO categories between different expressed and other nearest-neighbor genes of ER binding sites (number of genes $\geq 100$ ).

\begin{tabular}{llll}
\hline Genes set & Biological process & Molecular function & Cellular component \\
\hline \multirow{2}{*}{ Differently expressed } & $\begin{array}{l}\text { Biological regulation, metabolic process, } \\
\text { cell communication, organismal process, } \\
\text { localization, developmental process } \\
\text { Others }\end{array}$ & $\begin{array}{l}\text { Protein binding, } \\
\text { iron binding }\end{array}$ & $\begin{array}{l}\text { Membrane, } \\
\text { nucleus }\end{array}$ \\
\hline
\end{tabular}

TABLE 6: KEGG pathways enriched with the nearest-neighbor genes of ER binding sites $(P$-value $<0.01)$.

\begin{tabular}{llccc}
\hline KEGG ID & Pathways name & $P$-value & Number of genes & Number of different expressed genes \\
\hline hsa05200 & Pathways in cancer & $2.24 E-05$ & 22 & 16 \\
hsa04510 & Focal adhesion & 0.0002 & 15 & 14 \\
hsa04360 & Axon guidance & 0.0009 & 11 & 8 \\
hsa04810 & Regulation of actin cytoskeleton & 0.0012 & 14 & 11 \\
hsa04010 & MAPK signaling pathway & 0.0022 & 15 & 12 \\
hsa04114 & Oocyte meiosis & 0.0024 & 9 & 8 \\
hsa04144 & Endocytosis & 0.0024 & 12 & 11 \\
hsa04115 & p53 signaling pathway & 0.0024 & 7 & 7 \\
hsa05216 & Thyroid cancer & 0.0024 & 5 & 4 \\
hsa05218 & Melanoma & 0.0033 & 7 & 3 \\
hsa04020 & Calcium signaling pathway & 0.004 & 11 & 4 \\
hsa04062 & Chemokine signaling pathway & 0.0064 & 11 & 9 \\
hsa04914 & Progesterone-mediated oocyte maturation & 0.0085 & 7 & 7 \\
hsa01100 & Metabolic pathways & 0.0086 & 35 & 28 \\
hsa00450 & Selenoamino acid metabolism & 0.0088 & 4 & 7 \\
hsa05414 & Dilated cardiomyopathy & 0.0096 & 7 & 3 \\
hsa03440 & Homologous recombination & 0.0097 & 4 & 3 \\
\hline
\end{tabular}

TABle 7: Terms of the enriched GeneGo pathway maps $(P$-value $<0.01$, FDR $<0.05)$.

\begin{tabular}{lc}
\hline GeneGo pathway terms & $P$-value \\
\hline Apoptosis and survival_APRIL and BAFF signaling & $1.29889 E-05$ \\
Development_prolactin receptor signaling & $4.95517 E-05$ \\
Development_glucocorticoid receptor signaling & $5.81237 E-05$ \\
Development_ligand -independent activation of ESR1 and ESR2 & 0.000295251 \\
Immune response_IL-22 signaling pathway & 0.000381484 \\
Development_EPO-induced Jak-STAT pathway & 0.000531744 \\
Development_growth hormone signaling via STATs and PLC/IP3 & 0.000531744 \\
Cytoskeleton remodeling_keratin filaments & 0.000622315 \\
Development_GM-CSF signaling & 0.000660576 \\
Transcription_transcription regulation of aminoacid metabolism & 0.000752764 \\
\hline
\end{tabular}

relationship with ER. APRIL and BAFF were the members of tumor necrosis factor family which related to a plethora of cellular events from proliferation and differentiation to apoptosis and tumor reduction [42]. IL-22 might play a role in the control of tumor growth and progression in breast [43]. However, the relationship between ER and these two pathways need further experimental study.

\section{Conclusions}

ER is an important molecular symbol of breast cancer. A full understanding of the molecular mechanisms of ER will be useful for the research in the prediction and treatment of breast cancer. The ChIP-Seq technology is useful to study the interaction of protein and DNA on a genome-wide scale. ChIP-Seq data can effectively analyze the regulatory mechanism of transcription factor in genome-wide scale. In this study, we used ChIP-Seq data to identify the global sites regulated by ER in MCF-7 breast cancer cell line. In order to get more reliable result, three different tools were used to analyze two datasets. And 933 binding sites were identified, and the ERE motif was refined here.

The analysis of the global genomic occupancy of ERregulated genes revealed that $92 \%$ of the total 933 ER-binding 


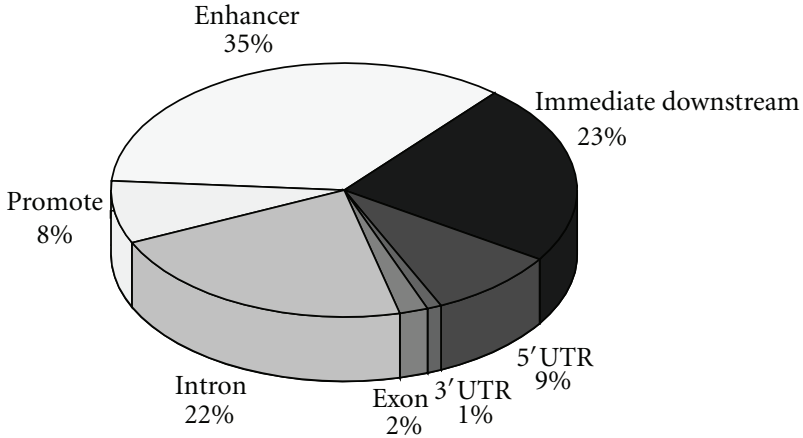

(a)

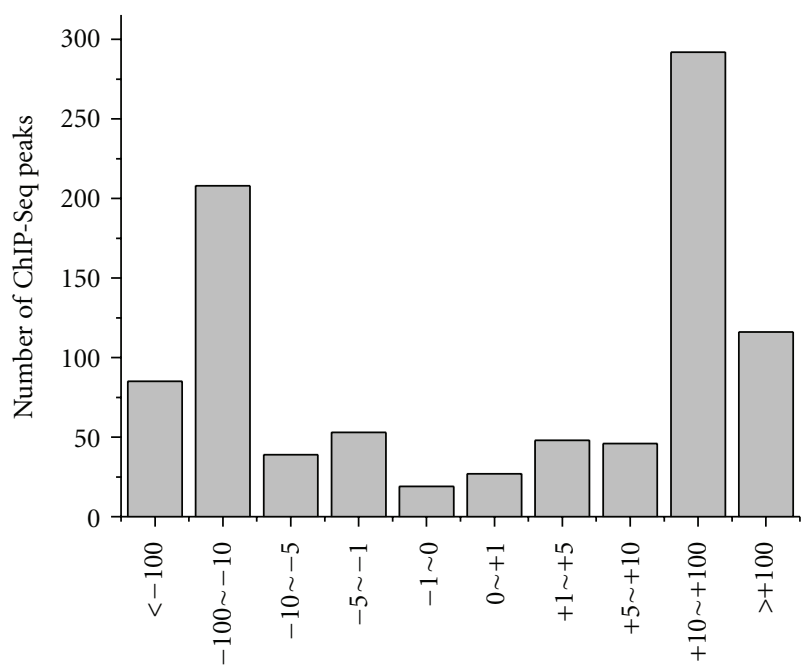

ER ChIP-Seq peak location $(\mathrm{kb})$

(b)

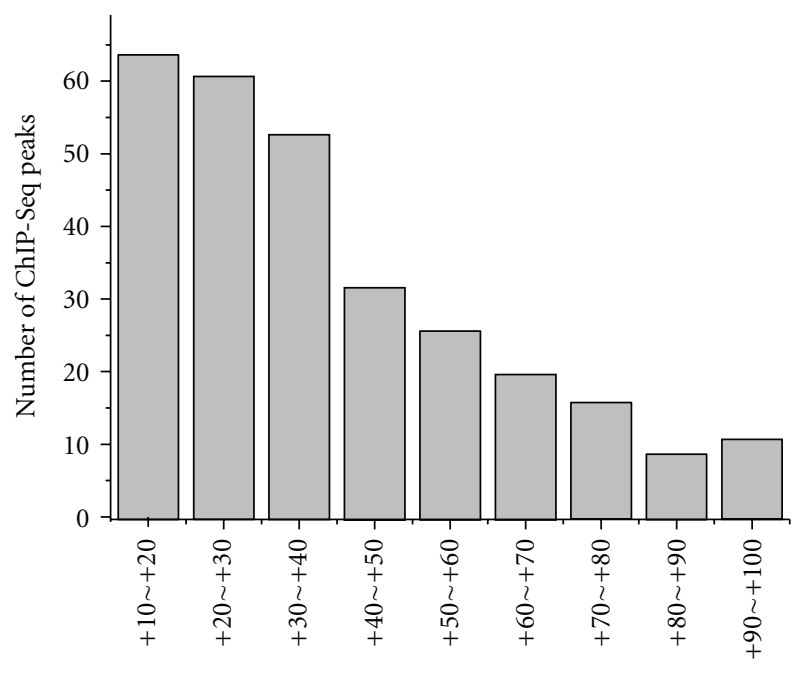

ER ChIP-Seq peak location $(\mathrm{kb})$

(c)

FIGURE 4: Location analysis of ER binding sites. (a) locations relative to nearest-neighbor genes. (b) Genomic Locations of ER ChIP-Seq peaks. (c) Genomic locations of ER ChIP-Seq peaks within $+10 \sim$ $+100 \mathrm{~kb}$.

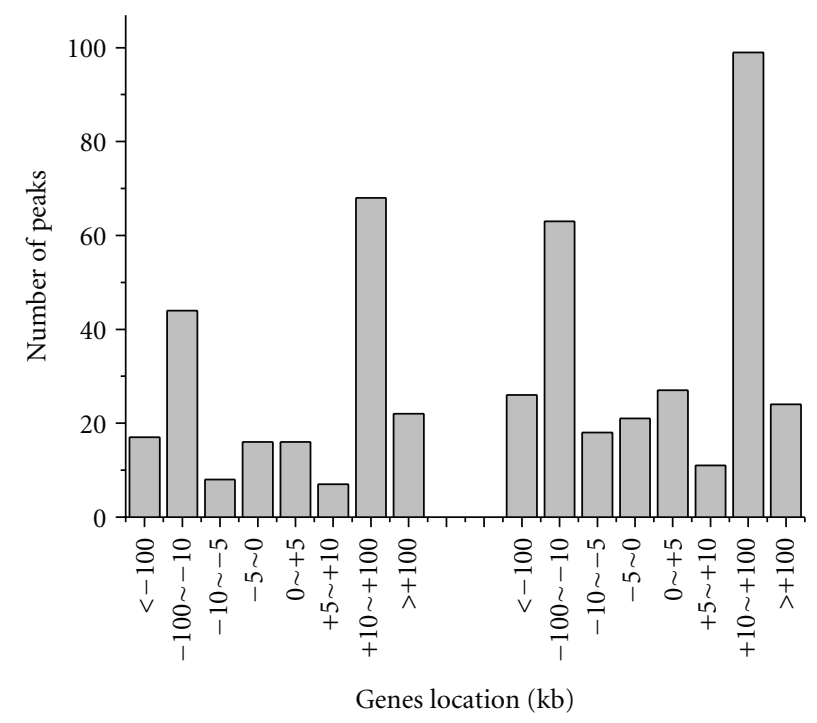

FIGURE 5: Genomic Locations of differentially expressed genes in the vicinity of ER binding sites.

sites were located far away from promoters. This suggested that the canonical mode of ER factor function involved longrange control. Previous research had reported that ER- $\alpha$ includes looping [44]. Using ChIP-PET, Lin et al. [27] had analyzed the genome-wide ER- $\alpha$ chromatin occupancy and revealed abundant nonpromoter sites. Our findings provided further support for this mode of ER factor function.

We compared the ER binding sites found in this study with published differentially expressed genes between ER+ and ER- breast tumors. A set of 487 genes was found significant in discriminating ER status in breast tumors. This indicated that these genes appeared to affect ER response. Only 9\% (44) of the genes have been identified by Lin et al. [27], while the remaining need further validations. We found that binding sites were preferentially associated with ER upregulated genes, indicating that ER was more frequently involved in the regulation of upregulated genes. The location of 487 genes verified the long-range control mode of ER factor.

In this study, we found 2694 single nucleotide polymorphisms loci located in or near the ER binding sites. Among these SNPs, the 157 genes of 836 SNPs were also differentially expressed between ER+ and ER- breast tumors. It indicated that this set of SNPs might have close relationship with ER in breast.

The functional annotation provided a deeper understanding of ER and ER-associated genes. Enrichment analysis of GO gave an overview of gene function. As shown in Figure 6, significantly enriched terms belonged to three classes, biological regulation, cellular processes, and developmental processes. The result of KEGG enrichment analysis was similar. Five pathways were involved in cellular processes, including focal adhesion, regulation of actin cytoskeleton, oocyte meiosis, endocytosis, and p53 signaling pathway. These pathways were associated with cell communication, 


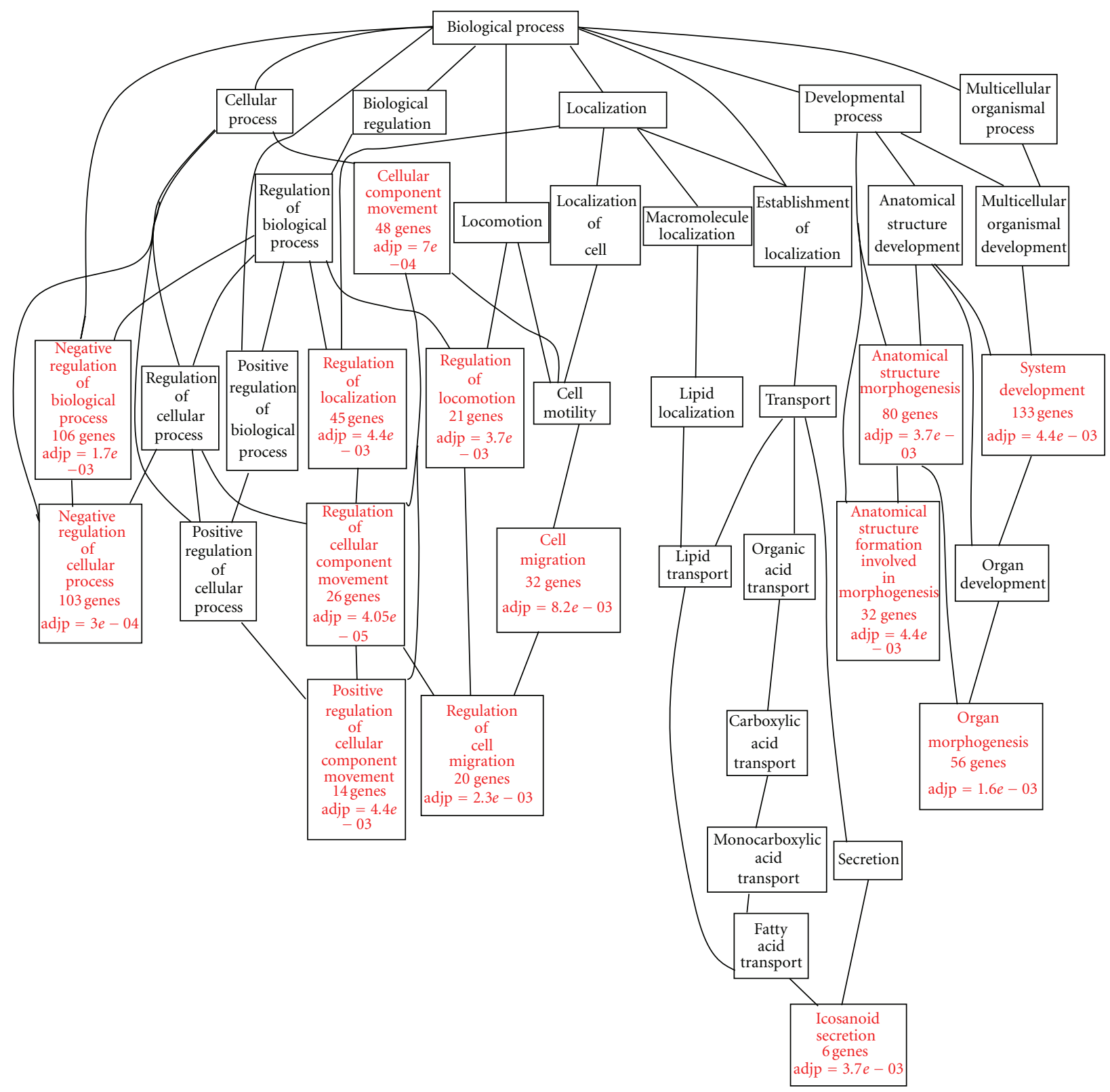

Figure 6: Directed Acyclic Graphs (DAGs) of significantly enriched GO (Gene Ontology) categories $(P<0.01)$.

movement, growth, and death. Most enriched terms determined by GeneGO were development pathways. It was suggested that ER-regulated genes participated in various development processes. Moreover, KEGG pathway analysis suggested that ER-regulated genes were enriched in some diseases related pathways. Both KEGG and GeneGO pathway analysis revealed that some immune-related pathways were enriched, such as chemokine signaling pathway and immune response_IL-22 signaling pathway. These results indicated that ER-regulated genes related to the development, progression, and metastasis of breast. ER affected every developed stage of breast. However, the regulatory mechanisms of ER in different stages and different pathways still need further studies.

\section{Conflict of Interests}

The authors declare that they have no conflict of interests.

\section{Acknowledgments}

This work was supported by the National Natural Science Foundation of China Grants (no. 91230117 and 31170795), the Specialized Research Fund for the Doctoral Program of Higher Education of China (20113201110015), International S\&T Cooperation Program of Suzhou (SH201120), and the National High Technology Research and Development Program of China (863 program, Grant no. 2012AA02A601). 


\section{References}

[1] M. J. van de Vijver, Y. D. He, L. J. van'T Veer et al., "A gene-expression signature as a predictor of survival in breast cancer," The New England Journal of Medicine, vol. 347, no. 25, pp. 1999-2009, 2002.

[2] M. A. Lopez-Garcia, F. C. Geyer, M. Lacroix-Triki, C. Marchió, and J. S. Reis-Filho, "Breast cancer precursors revisited: molecular features and progression pathways," Histopathology, vol. 57, no. 2, pp. 171-192, 2010.

[3] W. F. Anderson, N. Chatterjee, W. B. Ershler, and O. W. Brawley, "Estrogen receptor breast cancer phenotypes in the surveillance, epidemiology, and end results database," Breast Cancer Research and Treatment, vol. 76, no. 1, pp. 27-36, 2002.

[4] S. Mandal and J. R. Davie, "An integrated analysis of genes and pathways exhibiting metabolic differences between estrogen receptor positive breast cancer cells," BMC Cancer, vol. 7, article 181, 2007.

[5] M. C. Abba, Y. Hu, H. Sun et al., "Gene expression signature of estrogen receptor $\alpha$ status in breast cancer," BMC Genomics, vol. 6, no. 1, article 37, 2005.

[6] M. Hu, J. Yu, J. M. G. Taylor, A. M. Chinnaiyan, and Z. S. Qin, "On the detection and refinement of transcription factor binding sites using ChIP-Seq data," Nucleic Acids Research, vol. 38, no. 7, Article ID gkp1180, pp. 2154-2167, 2010.

[7] W. J. Welboren, M. A. van Driel, E. M. Janssen-Megens et al., "ChIP-Seq of ER $\alpha$ and RNA polymerase II defines genes differentially responding to ligands," EMBO Journal, vol. 28, no. 10, pp. 1418-1428, 2009.

[8] B. Langmead, C. Trapnell, M. Pop, and S. L. Salzberg, "Ultrafast and memory-efficient alignment of short DNA sequences to the human genome," Genome Biology, vol. 10, no. 3, article R25, 2009.

[9] A. P. Fejes, G. Robertson, M. Bilenky, R. Varhol, M. Bainbridge, and S. J. M. Jones, "FindPeaks 3.1: a tool for identifying areas of enrichment from massively parallel shortread sequencing technology," Bioinformatics, vol. 24, no. 15, pp. 1729-1730, 2008.

[10] A. P. Boyle, J. Guinney, G. E. Crawford, and T. S. Furey, "FSeq: a feature density estimator for high-throughput sequence tags," Bioinformatics, vol. 24, no. 21, pp. 2537-2538, 2008.

[11] H. Ji, H. Jiang, W. Ma, D. S. Johnson, R. M. Myers, and W. H. Wong, "An integrated software system for analyzing ChIP-chip and ChIP-seq data," Nature Biotechnology, vol. 26, no. 11, pp. 1293-1300, 2008.

[12] Y. Zhang, T. Liu, C. A. Meyer et al., "Model-based analysis of ChIP-Seq (MACS)," Genome Biology, vol. 9, no. 9, article R137, 2008.

[13] R. Jothi, S. Cuddapah, A. Barski, K. Cui, and K. Zhao, "Genome-wide identification of in vivo protein-DNA binding sites from ChIP-Seq data," Nucleic Acids Research, vol. 36, no. 16, pp. 5221-5231, 2008.

[14] A. Valouev, D. S. Johnson, A. Sundquist et al., "Genome-wide analysis of transcription factor binding sites based on ChIPSeq data," Nature Methods, vol. 5, no. 9, pp. 829-834, 2008.

[15] R. Redon, S. Ishikawa, K. R. Fitch et al., "Global variation in copy number in the human genome," Nature, vol. 444, no. 7118, pp. 444-454, 2006.

[16] T. L. Bailey and C. Elkan, "Fitting a mixture model by expectation maximization to discover motifs in biopolymers," in Proceedings of the International Conference on Intelligent Systems for Molecular Biology, vol. 2, pp. 28-36, 1994.

[17] J. Li and R. Tibshirani, "Finding consistent patterns: a nonparametric approach for identifying differential expression in
RNA-Seq data," Statistical Methods in Medical Research. In press.

[18] V. G. Tusher, R. Tibshirani, and G. Chu, "Significance analysis of microarrays applied to the ionizing radiation response," Proceedings of the National Academy of Sciences of the United States of America, vol. 98, no. 9, pp. 5116-5121, 2001.

[19] S. T. Sherry, M. H. Ward, M. Kholodov et al., "DbSNP: the NCBI database of genetic variation," Nucleic Acids Research, vol. 29, no. 1, pp. 308-311, 2001.

[20] M. Ashburner, C. A. Ball, J. A. Blake et al., "Gene ontology: tool for the unification of biology," Nature Genetics, vol. 25, no. 1, pp. 25-29, 2000.

[21] M. Kanehisa and S. Goto, "KEGG: kyoto encyclopedia of genes and genomes," Nucleic Acids Research, vol. 28, no. 1, pp. 27-30, 2000.

[22] B. Zhang, D. Schmoyer, S. Kirov, and J. Snoddy, "GOTree Machine (GOTM): a web-based platform for interpreting sets of interesting genes using gene ontology hierarchies," BMC Bioinformatics, vol. 5, article 16, 2004.

[23] B. Zhang, S. Kirov, and J. Snoddy, "WebGestalt: an integrated system for exploring gene sets in various biological contexts," Nucleic Acids Research, vol. 33, no. 2, pp. W741-W748, 2005.

[24] K. Graham, X. Ge, A. de Las Morenas, A. Tripathi, and C. L. Rosenberg, "Gene expression profiles of estrogen receptorpositive and estrogen receptor-negative breast cancers are detectable in histologically normal breast epithelium," Clinical Cancer Research, vol. 17, no. 2, pp. 236-246, 2011.

[25] Y. Wang, J. G. M. Klijn, Y. Zhang et al., "Gene-expression profiles to predict distant metastasis of lymph-node-negative primary breast cancer," The Lancet, vol. 365, no. 9460, pp. 671679, 2005.

[26] B. Lu, X. Liang, G. K. Scott et al., "Polyamine inhibition of estrogen receptor (ER) DNA-binding and ligand-binding functions," Breast Cancer Research and Treatment, vol. 48, no. 3, pp. 243-257, 1998.

[27] C. Y. Lin, V. B. Vega, J. S. Thomsen et al., "Whole-genome cartography of estrogen receptor $\alpha$ binding sites," PLoS Genetics, vol. 3, no. 6, article e87, 2007.

[28] A. Beeghly-Fadiel, W. Zheng, W. Lu et al., "Replication study for reported SNP associations with breast cancer survival," Journal of Cancer Research and Clinical Oncology, vol. 138, no. 6, pp. 1019-1026, 2012.

[29] W. Han, K. Y. Kim, S. J. Yang, D. Y. Noh, D. Kang, and K. Kwack, "SNP-SNP interactions between DNA repair genes were associated with breast cancer risk in a Korean population," Cancer, vol. 118, no. 3, pp. 594-602, 2012.

[30] C. H. Yang, L. Y. Chuang, Y. J. Chen, H. F. Tseng, and H. W. Chang, "Computational analysis of simulated SNP interactions between 26 growth factor-related genes in a breast cancer association study," OMICS A Journal of Integrative Biology, vol. 15, no. 6, pp. 399-407, 2011.

[31] K. D. Graves, B. N. Peshkin, G. Luta, W. Tuong, and M. D. Schwartz, "Interest in genetic testing for modest changes in breast cancer risk: implicationsfor SNP testing," Public Health Genomics, vol. 14, no. 3, pp. 178-189, 2011.

[32] R. J. Hartmaier, S. Tchatchou, A. S. Richter et al., "Nuclear receptor coregulator SNP discovery and impact on breast cancer risk," BMC Cancer, vol. 9, article 438, 2009.

[33] A. L. Lark, C. A. Livasy, L. Dressler et al., "High focal adhesion kinase expression in invasive breast carcinomas is associated with an aggressive phenotype," Modern Pathology, vol. 18, no. 10, pp. 1289-1294, 2005.

[34] A. S. Oh, L. A. Lorant, J. N. Holloway, D. L. Miller, F. G. Kern, and D. El-Ashry, "Hyperactivation of MAPK induces 
loss of ER $\alpha$ expression in breast cancer cells," Molecular Endocrinology, vol. 15, no. 8, pp. 1344-1359, 2001.

[35] H. Cam, H. Griesmann, M. Beitzinger et al., "p53 family members in myogenic differentiation and rhabdomyosarcoma development," Cancer Cell, vol. 10, no. 4, pp. 281-293, 2006.

[36] M. P. DeYoung, C. M. Johannessen, C. O. Leong, W. Faquin, J. W. Rocco, and L. W. Ellisen, "Tumor-specific p73 upregulation mediates p63 dependence in squamous cell carcinoma," Cancer Research, vol. 66, no. 19, pp. 9362-9368, 2006.

[37] G. Dominguez, J. M. Silva, J. Silva et al., "Wild type p73 overexpression and high-grade malignancy in breast cancer," Breast Cancer Research and Treatment, vol. 66, no. 3, pp. 183190, 2001.

[38] A. Chédotal, "Chemotropic axon guidance molecules in tumorigenesis," Progress in Experimental Tumor Research, vol. 39, pp. 78-90, 2007.

[39] H. Yamaguchi and J. Condeelis, "Regulation of the actin cytoskeleton in cancer cell migration and invasion," Biochimica et Biophysica Acta, vol. 1773, no. 5, pp. 642-652, 2007.

[40] K. McHale, J. E. Tomaszewski, R. Puthiyaveettil, V. A. Livolsi, and C. V. Clevenger, "Altered expression of prolactin receptorassociated signaling proteins in human breast carcinoma," Modern Pathology, vol. 21, no. 5, pp. 565-571, 2008.

[41] P. Moutsatsou and A. G. Papavassiliou, "The glucocorticoid receptor signalling in breast cancer: breast carcinoma," Journal of Cellular and Molecular Medicine, vol. 12, no. 1, pp. 145-163, 2008.

[42] V. Pelekanou, M. Kampa, M. Kafousi et al., "Expression of TNF-superfamily members BAFF and APRIL in breast cancer: immunohistochemical study in 52 invasive ductal breast carcinomas," BMC Cancer, vol. 8, article 76, 2008.

[43] G. F. Weber, F. C. Gaertner, W. Erl et al., "IL-22-mediated tumor growth reduction correlates with inhibition of ERK1/2 and AKT phosphorylation and induction of cell cycle arrest in the G 2-M phase," Journal of Immunology, vol. 177, no. 11, pp. 8266-8272, 2006.

[44] J. S. Carroll, X. S. Liu, A. S. Brodsky et al., "Chromosomewide mapping of estrogen receptor binding reveals long-range regulation requiring the forkhead protein FoxA1," Cell, vol. 122, no. 1, pp. 33-43, 2005. 


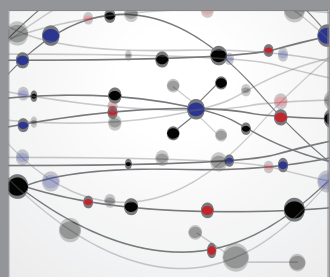

The Scientific World Journal
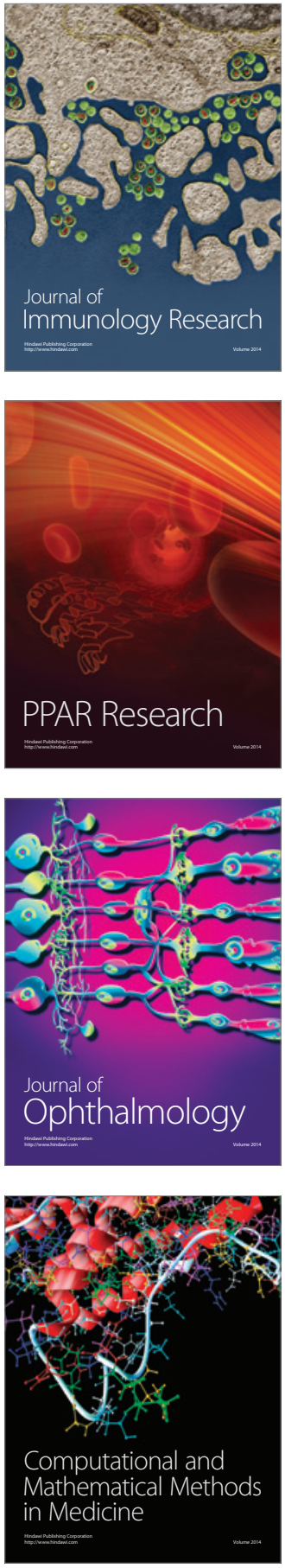

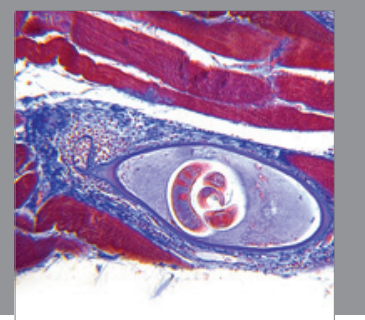

Gastroenterology

Research and Practice
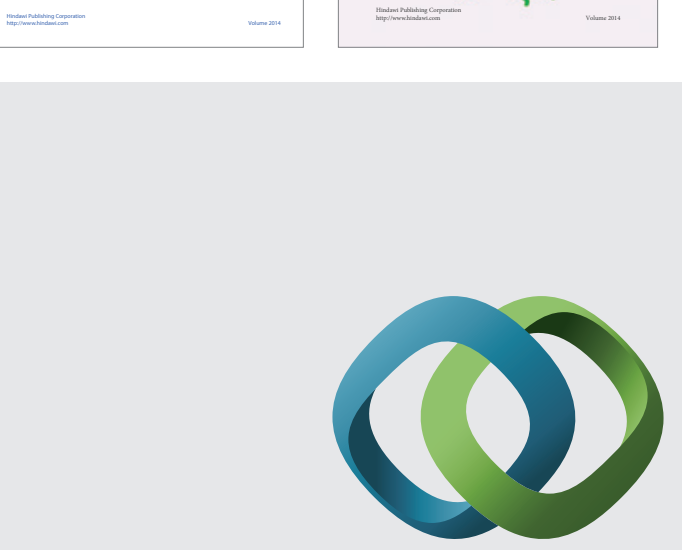

\section{Hindawi}

Submit your manuscripts at

http://www.hindawi.com
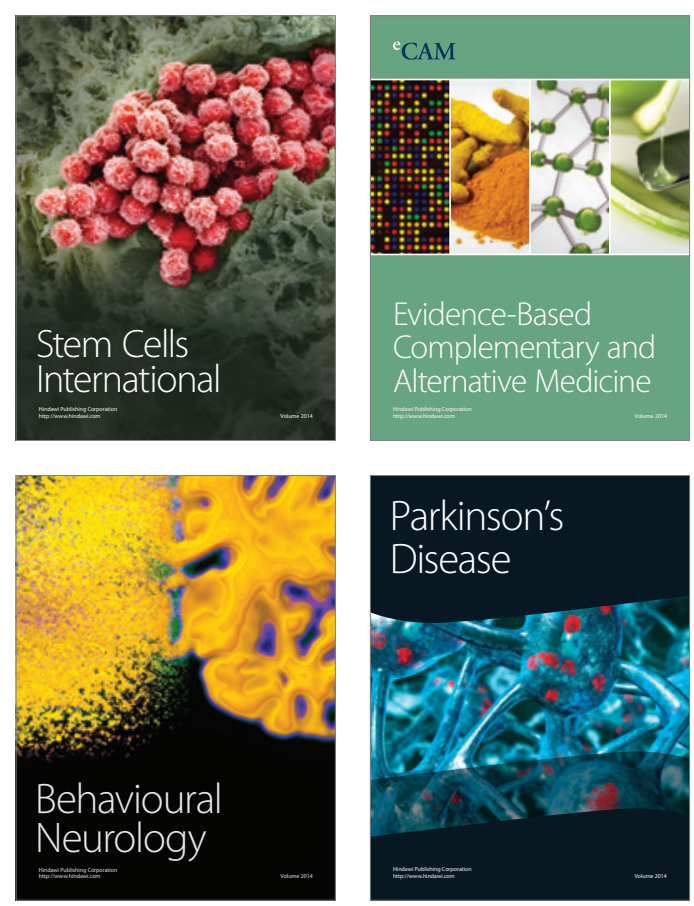

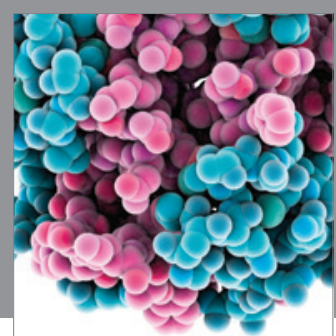

Journal of
Diabetes Research

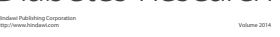

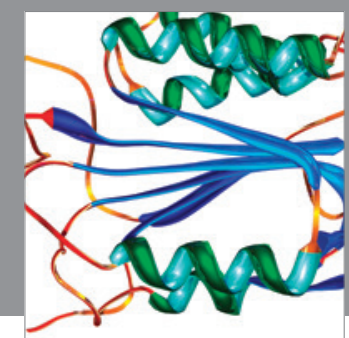

Disease Markers
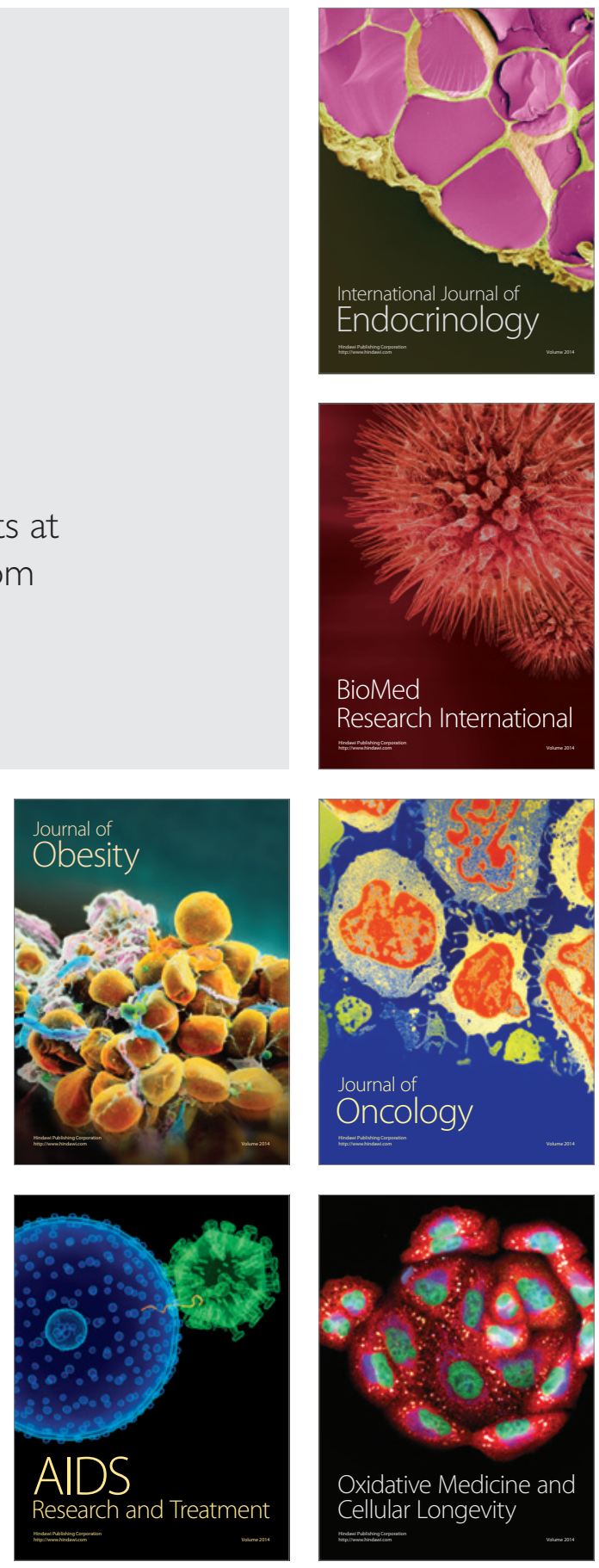